\title{
MODELAGEM MATEMÁTICA E LETRAMENTO CIENTÍFICO NO ENSINO DE FÍSICA
}

\author{
Ednilson Sergio Ramalho de Souzal \\ Adilson Oliveira do Espírito Santo2
}

\section{RESUMO}

$\mathrm{Na}$ atual sociedade tecnológica e globalizada, permeada de rápidas transformações socioculturais, os conteúdos para o ensino de física não podem mais se concentrar na aprendizagem conceitual. Além de conceitos, os estudantes precisam construir competências que os possibilitem investigar e tomar decisões críticas cientificamente fundamentadas, ou seja, é necessário letramento científico. Competências desse tipo envolvem a adoção de uma abordagem educacional ativa, tal como a modelagem matemática. O objetivo deste artigo é analisar a modelagem como geradora de competências essenciais para a formação de sujeitos letrados cientificamente no contexto amazônico do ensino de física. A pesquisa de campo envolveu três ciclos de modelagem com professores de ciências $(n=13)$ do município de Almeirim, Pará, Brasil. Os métodos para produção de dados foram inspirados na metodologia mista. A interpretação de informações recorreu a procedimentos da análise textual discursiva em três fases: unitarização, categorização e metatexto. Os resultados evidenciam que a modelagem matemática pode ter implicações positivas para o letramento científico em física, especialmente para desenvolver competências para diferenciar questões com possibilidades de serem investigadas cientificamente, para avaliar formas de explorar cientificamente dada questão e para usar diferentes linguagens visando a manifestar aprendizagens.

Palavras-chave: Modelagem matemática. Letramento científico. Ensino de física.

\section{MATHEMATICAL MODELING AND SCIENTIFIC LITERACY IN THE PHYISICS TEACHING}

\begin{abstract}
In today's technological and globalized society, permeated by rapid sociocultural transformations, content for physics teaching can no longer focus on conceptual

1 Doutor em Educação em Ciências e Matemática (UFMT). Professor do Instituto de Ciências da Educação (ICED) da Universidade Federal do Oeste do Pará (UFOPA). Orcid iD: https://orcid.org/0000-0002-2816-0941.E-mail: ednilson.souza@ufopa.edu.br

2 Doutor em Engenharia Elétrica (UNICAMP). Professor do Instituto de Educação Matemática e Científica (IEMCI) da Universidade Federal do Pará (UFPA). Orcid iD: https://orcid.org/00000003-2941-0786. E-mail: adilson@ufpa.br
\end{abstract}


learning. In addition to concepts, students need to build competencies that enable them to investigate and make critical decisions that are scientifically grounded, scientific literacy is needed. Competencies of this kind involve the adoption of an active educational approach, such as mathematical modeling. The purpose of this article is to analyze the modeling as the generator of essential skills for the training of scientifically literate subjects in the Amazonian context of physics teaching. Field research involved three modeling cycles with science teachers ( $n=13$ ) from the city of Almeirim, Pará, Brazil. The methods for producing data were inspired by the mixed methodology. The interpretation of information resorted to procedures of discursive textual analysis in three phases: unitarization, categorization and metatext. The results show that mathematical modeling can have positive implications for scientific literacy in physics, especially to develop competencies to differentiate issues with possibilities for scientific investigation, to evaluate ways to explore scientifically the issue and to use different languages in order to manifest learning.

Keywords: Mathematical modeling. Scientific literacy. Physics teaching.

\section{MODELACIÓN MATEMÁTICA Y ALFABETIZACIÓN CIENTÍFICA EN LA ENSEÑANZA DE LA FÍSICA}

\section{RESUMEN}

En la sociedad tecnológica globalizada de hoy, impregnada por rápidas transformaciones socioculturales, los contenidos para la enseñanza de la física ya no pueden centrarse en el aprendizaje conceptual. Además de los conceptos, los estudiantes necesitan desarrollar competencias que les permitan investigar y tomar decisiones científicamente informadas críticas, es decir, se requiere alfabetización científica. Las competencias de este tipo implican adoptar un enfoque educativo activo, como el modelación matemática. El objetivo de este trabajo es analizar el modelación como generador de competencias esenciales para la formación de sujetos con conocimientos científicos en el contexto amazónico de la enseñanza de la física. La investigación de campo incluyó tres ciclos de modelación con profesores de ciencias ( $n=13$ ) del municipio de Almeirim, Pará, Brasil. Los métodos para la producción de datos se inspiraron en la metodología mixta. La interpretación de la información recurrió a los procedimientos de análisis textual discursivo en tres fases: unitarización, categorización y metatexto. Los resultados muestran que el modelación matemática puede tener implicaciones positivas para la alfabetización científica en física, especialmente para desarrollar habilidades para diferenciar cuestiones que pueden investigarse científicamente, para evaluar formas de explorar científicamente una pregunta dada y para usar diferentes idiomas para expresar el aprendizaje.

Palabras-clave: Modelación matemática. Alfabetización científica. Enseñanza de la física.

\section{CONTORNO INICIAL}

Na sociedade tecnocientífica do emergente século XXI, de rápidas transformações socioculturais, causadas em certo grau pelo avanço da 
ciência e da tecnologia, os objetivos para o ensino de física não podem mais se concentrar na aprendizagem conceitual. Além de conceitos, os estudantes precisam construir competências ${ }^{3}$ que os possibilitem investigar e tomar decisões críticas cientificamente fundamentadas. Ou seja, é cada vez maior a necessidade de letramento científico ${ }^{4}$. Infelizmente, no Brasil, pesquisas (BRASIL, 2016) mostram que esse é um objetivo ainda distante, exigindo de nós, enquanto formadores de educadores, a proposição de abordagens educacionais que levem à formação desejada.

Nesse cenário, o baixo índice de estudantes com proficiência científica acima de dois pontos (próximo de 18\%), registrado na edição do PISA 2015 (BRASIL, 2016), coloca em dúvida a efetividade de aulas de física com foco na memorização e na mecanização de procedimentos, infelizmente, ainda comuns em muitas escolas brasileiras. Não se quer dizer que a memorização e a mecanização não sejam necessárias, mas defendese a necessidade de procurar alternativas ao chamado ensino bancário, no qual o professor apenas "deposita" informações na mente do estudante; este, em analogia a um cofre, dificilmente possui abertura para investigar, para questionar, para refletir sobre o objeto de aprendizagem (FREIRE, 2005).

Decerto que orientações para uma prática pedagógica em ciências da natureza que leve ao letramento científico não são novas nas normativas educacionais brasileiras. Nessa direção, na década de 1990 já se destacava como um dos objetivos básicos do ensino fundamental que os estudantes fossem capazes de analisar a realidade, de formular e de resolver problemas, de utilizar o pensamento lógico, de selecionar e de verificar procedimentos investigativos (BRASIL, 1997). Tal objetivo, dada sua importância para a

\footnotetext{
3 Ressalta-se que o termo "competência" está sendo usado no sentido atribuído por Perrenoud (2013), ou seja, tanto como produto de uma aprendizagem quanto fundamento da ação humana: "a competência é o poder de agir com eficácia em uma situação, mobilizando e combinando, em tempo real e de modo pertinente, os recursos intelectuais e emocionais (p. 45)".

$4 \mathrm{Em}$ todo o texto não se fará distinção entre os termos letramento científico, alfabetização científica, enculturação científica, literacia científica; embora alguns autores propõem tal distinção, será usado somente o termo letramento científico conforme a Base Nacional Comum Curricular (BRASIL, 2018).
} 
formação cidadã, também se constituiu como um dos objetivos principais do ensino de ciências da natureza no nível médio (BRASIL, 2006).

Desse modo, em consonância com as normativas para o ensino brasileiro de ciências da natureza, entende-se que buscar letramento científico em física seja importante, porque envolve capacidades para compreender e para interpretar situações do mundo natural, do mundo social, do mundo tecnológico; bem como para transformá-los com base nos aportes teóricos e processuais da própria ciência (BRASIL, 2017).

Nessa linha, deseja-se formar sujeitos com um mínimo de competências para investigação e para argumentação científica sobre situações reais que envolvem complexas relações do mundo em que vivem. A modelagem matemática, assim se propõe neste artigo, pode ser uma abordagem educacional que vai ao encontro dessa formação.

Apesar de na última década a literatura brasileira sobre ensino de física apresentar diversas pesquisas com foco em modelagem matemática, para citar algumas: Barsotti (2013); Brandão, Araújo e Veit (2008); Batista (2009); Batista e Kato (2008); Heidemann (2015); Heidemann, Araújo e Veit (2016); Heidemann, Araújo e Veit (2012); Lozada e Magalhães (2009); Maciel (2014); Monteiro (2013); Moutinho (2007); Mendes, Consta e Sousa (2012); Silva Neto (2015); Souza (2016); Souza (2012); Souza (2010); Souza e Espírito Santo (2017); Wolff e Serrano (2011), verifica-se que poucas são as que se destinam a investigar sistematicamente possibilidades para o letramento científico. Assim, o objetivo principal é analisar a modelagem matemática como geradora de competências essenciais para a formação de sujeitos letrados cientificamente no contexto amazônico do ensino de física.

Com base em tal objetivo, realizaram-se três ciclos de modelagem com professores de ciências $(n=13)$ da rede de educação municipal da cidade de Almeirim-PA, Brasil. Os métodos para analisar os ciclos de modelagem foram inspirados na metodologia mista, com procedimentos tanto qualitativos, quanto quantitativos (CRESWELL e CLARK, 2013). Nesse direcionamento, houve aplicação de questionários fechados cujas respostas 
foram interpretadas por meio de análise textual discursiva (MORAES e GALIAZZI, 2016).

Na seção que segue, discute-se sobre a ideia de letramento científico e sobre competências essenciais apresentadas na literatura. Na segunda seção, o foco será sobre o ciclo de modelagem de David Hestenes 5 (HEIDEMANN, 2015) como método importante de sala de aula. Na terceira seção, apresenta-se a metodologia da pesquisa de campo numa abordagem mista. Na quarta seção, o olhar estará voltado às competências para o letramento científico identificadas pelos sujeitos da pesquisa nos ciclos de modelagem desenvolvidos. Finaliza-se com implicações do estudo para o ensino de física, especialmente no contexto amazônico.

\section{ENSINO DE FÍSICA PARA ALÉM DE CONCEITOS}

Letramento científico, termo que se traduz da língua inglesa: scientific literacy, tem sido um objetivo para o ensino de ciências da natureza almejado por diversos países desde o século passado. Ganhou força após o lançamento do satélite Sputnik pela antiga Rússia, em outubro de 1957, o que chamou a atenção dos Estados Unidos da América para o fato de que deveriam investir na educação científica e tecnológica dos jovens estadunidenses (SOUZA, 2018). Mas o que entender por letramento científico?

A atual Base Nacional Comum Curricular considera que o letramento científico "envolve a capacidade de compreender e interpretar o mundo (natural, social e tecnológico), mas também de transformá-lo com base nos aportes teóricos e processuais das ciências" (BRASIL, 2018, p. 321). Ou seja, o estudante letrado cientificamente é um sujeito que possui competências fundamentais para propor justificativas científicas sobre o mundo em que vive, podendo modificá-lo para melhorar sua condição e da comunidade ao redor.

\footnotetext{
${ }^{5}$ Físico e educador norteamericano que, de acordo com a American Modeling Teachers Association (AMTA), atualmente tem se destacado como um dos grandes colaboradores para a reformulação do ensino de ciências estadunidense.
} 
Para o químico e educador Attico Chassot seria um "[...] conjunto de conhecimentos que facilitaria aos homens e mulheres fazer uma leitura do mundo onde vivem" (CHASSOT, 2014a, p. 62). Apesar de importante ao apontar que letramento científico (chamado pelo autor de alfabetização científica) se relaciona à leitura do mundo vivencial, essa concepção, dada sua amplitude, dificilmente informa sobre habilidades ou características específicas desses conhecimentos que poderiam facilitar aos homens e às mulheres leitura do mundo onde estão inseridos.

Apoiando-se nas ideias de Paulo Freire, Sasseron e Machado (2017) entendem que o letramento científico se refere ao ensino de ciências,

[...] cujo objetivo é a formação do indivíduo que o permita resolver problemas de seu dia a dia, levando em conta os saberes próprios das ciências e as metodologias de construção de conhecimento próprias do campo científico (p. 11).

Para esses autores, a letramento científico deve levar à formação cidadã dos estudantes para o domínio funcional dos conhecimentos científicos e seus desdobramentos nas diferentes fases de suas vidas. Considera-se tal concepção interessante porque destaca a necessidade de três competências gerais que podem potencializar o desenvolvimento de cidadãos alfabetizados cientificamente, são elas: a compreensão de termos, de conceitos e de conhecimentos científicos fundamentais; a compreensão da natureza das ciências e dos fatores éticos e políticos que circundam sua prática e o entendimento das relações existentes entre ciência; tecnologia, sociedade e meio ambiente (SASSERON e MACHADO, 2017).

Nessa mesma direção, a Organização para Cooperação e Desenvolvimento Econômico (OCDE) considera que o letramento científico envolve a compreensão e o engajamento em discussões críticas sobre ciência e tecnologia. Para isso, a OCDE assevera que é necessário o domínio de três competências gerais: explicar cientificamente fenômenos da natureza, avaliar e planejar investigações científicas e interpretar cientificamente dados e evidências (OCDE, 2016). 
Nota-se que as competências gerais apontadas pela OCDE são similares às propostas por Sasseron e Machado (2017). A primeira competência abrange conhecimentos para fornecer explicações sobre fenômenos naturais, sobre artefatos e sobre tecnologias; bem como suas implicações para a sociedade. Requer, portanto, saberes sobre ideias fundamentais da ciência e sobre questões que moldem sua prática e seus objetivos. A segunda competência abrange o conhecimento e a compreensão da investigação científica para identificar questões que podem ser respondidas pela pesquisa científica, para identificar procedimentos apropriados que devem ser utilizados e para propor caminhos visando a responder questões levantadas. A terceira competência envolve interpretar e avaliar cientificamente dados e evidências; bem como analisar se as conclusões são justificáveis.

Embora haja na literatura pertinente uma diversidade de entendimentos sobre o que seja letramento científico, algumas vezes também chamado de alfabetização científica, enculturação científica ou literacia científica, pode-se entendê-lo como um objetivo educacional que visa a favorer aos estudantes um mínimo de argumentação científica sobre problemas da realidade, movendo-os de uma esfera inicial para uma esfera mais avançada de análise crítica e de propostas de soluções a problemas socioculturais. Nessa linha, ele envolve visão holística dos problemas que se apresentam no cotidiano, envolve discussões amplas e profundas sobre o papel da ciência e da tecnologia como modificadores das condições socioculturais e ambientais (SOUZA, 2018). Para tal envergadura, algumas competências fundamentais são necessárias.

\section{UM INVENTÁRIO DE COMPETÊNCIAS}

A literatura sobre letramento científico apresenta conjuntos de competências consideradas necessárias à formação de sujeitos para o domínio e para o uso dos conhecimentos científicos nos diferentes estágios de suas vidas. Nesse sentido, Sasseron e Machado (2017) propõem dez capacidades fundamentais que foram fruto de análises de atividades 
investigativas em situação normal de sala de aula, são elas: seriação, organização e classificação de informações; raciocínio lógico e raciocínio proporcional; levantamento e teste de hipóteses; justificativa, previsão e explicação. Nota-se que tais capacidades são inerentes ao processo de investigação em ciências da natureza.

Em revisão de literatura, Pizarro e Lopes Junior (2015) fizeram levantamento bibliográfico em doze periódicos nacionais e internacionais sobre ensino de ciências. Os autores sistematizaram oito capacidades essenciais necessárias ao desenvolvimento de estudantes cientificamente letrados: articulação de ideias; investigação; argumentação; leitura e escrita em ciências; problematização; criação e atuação. Essas competências complementam as de Sasseron e Machado (2017) ao voltarem o olhar para a formação do pensamento reflexivo do estudante, especialmente na dimensão crítico social. São importantes, portanto, na medida em que podem mediar a tomada de consciência sobre as implicações da ciência na vida das pessoas.

Por fim, a edição do PISA 2015 (BRASIL, 2016) apresentou um conjunto de três capacidades principais para investigar o nível de letramento científico de estudantes brasileiros, são elas: explicação de fenômenos científicos, avaliação e planejamento de investigações científicas, interpretação de dados e de evidências científicas. Frisa-se que, em conjunto, essas competências envolvem o conhecimento de conteúdo de física, o conhecimento procedimental e o conhecimento epistemológico sobre física.

Sublinha-se que as competências acima não são exaustivas, decerto que outras capacidades são apontadas por demais pesquisadores da área. Contudo, chama-se a atenção que possuem significados semelhantes, embora diferentes em suas formas de apresentação. Ao reagrupá-las a partir de seus significados convergentes, é possível construir um importante inventário de competências ao letramento científico, conforme o Quadro 1. $\mathrm{Na}$ coluna da esquerda, os códigos identificam vinte (20) competências organizadas sem pretensão hierárquica em ordem alfabética por meio de 
marcador alfanumérico. Na coluna da direita, as competências são caracterizadas por meio de frases que sintetizam seus significados.

QUADRO 1 - Inventário de competências ao letramento científico.

\begin{tabular}{|c|c|}
\hline Código & Competências \\
\hline C1 & Adquirir habilidades de comunicação oral e escrita. \\
\hline C2 & Ampliar a percepção social sobre a influência da ciência no cotidiano. \\
\hline C3 & Analisar, interpretar dados e tirar conclusões apropriadas. \\
\hline C4 & Avaliar argumentos científicos e evidências de diferentes fontes. \\
\hline C5 & Avaliar formas de explorar cientificamente dada questão. \\
\hline C6 & Avaliar os vários caminhos que os cientistas usam para assegurar a confiabilidade \\
dos dados.
\end{tabular}

Fonte: Autor (2018).

O inventário no Quadro 1 pode ser útil para orientar planejamentos pedagógicos e/ou análises de atividades didáticas visando a favorecer o desenvolvimento de sujeitos críticos e proativos em ambiente gerado pela modelagem matemática. Para isso, pode ser utilizado de duas maneiras distintas e complementares: de um lado, o pesquisador pode avaliar episódios de sala de aula e identificar possíveis competências emergentes nas falas e nas ações realizadas pelos estudantes. Essa maneira solicita a adoção de técnicas de análise de discurso e/ou análise de conteúdo.

Outra maneira é aplicar o inventário em forma de questionário fechado. Nesse caso, os próprios sujeitos a partir de suas percepções identificam possíveis competências nas tarefas realizadas. No entanto, para responder adequadamente, os sujeitos devem ter clareza do significado de 
cada competência presente no inventário. Utilizou-se na presente pesquisa essa segunda maneira para analisar ciclos de modelagem.

\section{O CICLO DE MODELAGEM DE HESTENES}

Decerto que na literatura sobre pesquisa em ensino de física existem diversas didáticas interessantes em modelagem matemática. Contudo, dois fatores contribuíram fortemente para a escolha do ciclo de modelagem de David Hestenes na presente pesquisa. Um deles é que a didática hestenesiana sustenta a importância do uso coletivo de múltiplas ferramentas de representação e de comunicação como importante para a reformulação de modelos mentais inconsistentes sobre o mundo real. Outro fator é que se trata de uma didática que focaliza a argumentação científica entre grupos colaborativos de estudantes durante a elaboração e a implementação de modelos matemáticos.

De maneira geral, o ciclo de modelagem de Hestenes pode ser organizado em dois estágios principais: elaboração do modelo e implementação do modelo, conforme estruturado na Figura 1:

FIGURA 1 - Uma estrutura para o ciclo de modelagem de Hestenes.

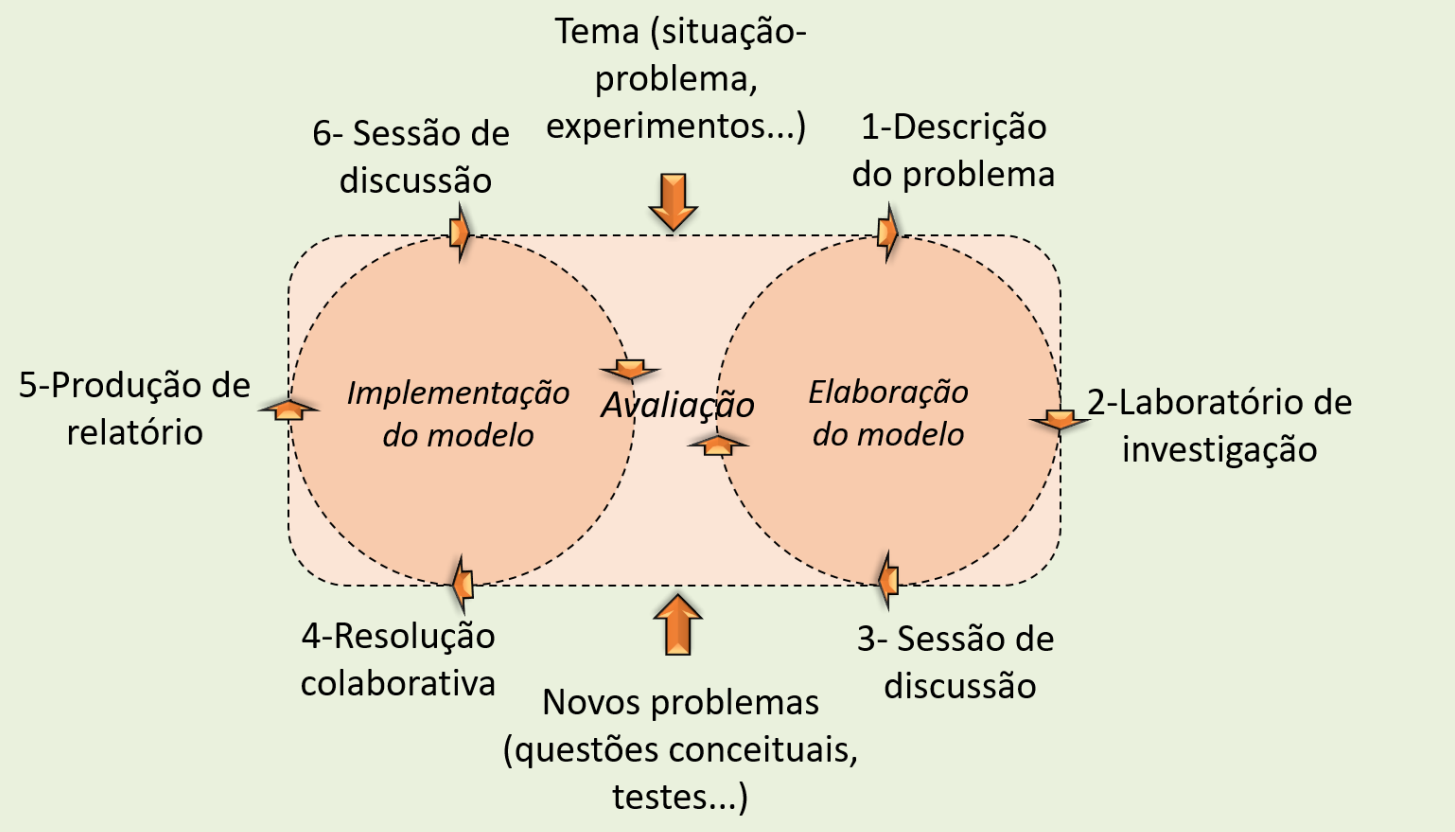

Fonte: Souza (2018). 


\section{Elaboração do modelo}

De acordo com o esquematizado na Figura 1, o primeiro estágio do ciclo de modelagem de Hestenes, elaboração do modelo, inicia com a descrição de um problema relacionado a algum tema de interesse dos estudantes. O problema pode ser proposto pelo professor ou pelos grupos de estudantes. O importante é que sejam atendidos tanto os objetivos pedagógicos do professor quanto os interesses dos estudantes pelo tema de pesquisa.

O objetivo principal da descrição do problema no ciclo de modelagem de Hestenes é evidenciar princípios, leis, teorias, variáveis, constantes; ou seja, grandezas físicas que se inter-relacionam no contexto da situação modelada. Hestenes (1987) argumenta que a descrição de um sistema é norteada por alguma teoria científica, pois é a teoria que especifica quais tipos de objetos e de propriedades podem ser modelados e quais tipos de modelos podem ser desenvolvidos.

Na sala de aula, algumas perguntas importantes surgem para nortear a descrição de um problema: quais quantidades ou grandezas estão variando na situação? Quais quantidades não estão variando? Quais quantidades afetam outras características? A finalidade dessas perguntas é levar os estudantes a perceberem as relações intrínsecas e extrínsecas ao sistema modelado, de modo a passarem naturalmente para a próxima etapa da elaboração do modelo.

Para iniciar a etapa de laboratório de investigação, a classe é organizada em grupos pequenos de três a cinco componentes (chamados grupos colaborativos). Ressalta-se que a palavra laboratório não deve ser entendida como um ambiente fechado cheio de equipamentos técnicos especializados, mas um momento em que serão planejadas e realizadas atividades que envolverão a observação de campo, a experimentação, a prática de determinada arte ou habilidade, os erros e os acertos.

Heidemann (2015) chama atenção que, na etapa de investigação laboratorial, os estudantes se envolvem em experimentações para resolver o problema proposto. Conforme a necessidade dos grupos, o professor 
apresenta novas ferramentas de representação (equações, gráficos, tabelas, diagramas etc.). Essas ferramentas são importantes no ciclo de modelagem porque a habilidade de modelar, portanto, de compreender, depende das ferramentas de representação disponíveis.

O produto do laboratório de investigação é um conjunto de representações simbólicas que devem ser logicamente coordenadas em um modelo matemático, fazendo-se uso de múltiplas ferramentas de representações e de comunicação. Para isso, são de fundamental importância os chamados whiteboards, ou seja, pequenos quadros brancos de aproximadamente $80 \mathrm{~cm} \times 60 \mathrm{~cm}$, conforme figura que segue.

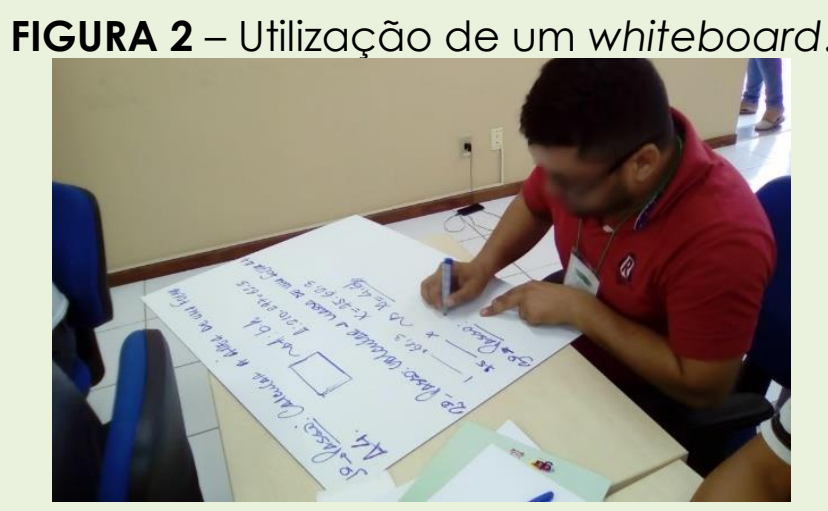

Fonte: Autor (2016).

Como ilustrado na Figura 2, cada whiteboard é utilizado para sistematizar por escrito um modelo matemático (ou parte dele) que será defendido e discutido coletivamente pelos grupos colaborativos. Importante deixar claro que, na visão de Hestenes (2006), a ideia de modelo matemático não se restringe a uma equação matemática. Um modelo matemático envolve uma visão holística relacionada às diversas ferramentas de representação que são utilizadas na solução de um problema e aos modelos mentais subjacentes aos raciocínios inerentes a tal resolução (SOUZA, 2018).

Continuando a descrição da Figura 1, na primeira sessão de discussão, as equipes apresentam seus modelos matemáticos e justificam procedimentos e raciocínios. Heidemann (2015) chama a atenção que, nesse momento, os grupos justificam suas conclusões de forma oral e escrita, 
enquanto que as ferramentas de representação destacadas nos whiteboards podem aprimorar a capacidade de argumentação dos estudantes.

Nesse processo argumentativo, conforme os modelos matemáticos vão sendo compartilhados pelos grupos colaborativos, as discussões sobre os pontos convergentes e os pontos divergentes permitem compreensão comum a respeito da estrutura epistêmica de um modelo geral de resolução. Tal estrutura epistêmica será aprofundada no segundo estágio do ciclo de modelagem de Hestenes: implementação do modelo.

\section{IMPLEMENTAÇÃO DO MODELO}

As atividades de implementação do modelo são importantes porque os estudantes aprofundam compreensões ao ramificarem a estrutura epistêmica do modelo geral na análise de aspectos específicos do fenômeno investigado. Hestenes (1987) sublinha que a ramificação é um processo principalmente matemático, por isso é importante para se trabalhar propriedades e implicações especiais do modelo matemático.

Conforme esquematizado na Figura 1, a implementação do modelo inicia com a resolução colaborativa de novos problemas. Problemas esses que suscitem a estrutura epistêmica do modelo matemático em foco. Nesse sentido, podem-se utilizar problemas adaptados do livro-texto, testes de múltipla escolha, questões conceituais, experimentos de baixo custo ou de laboratório técnico, simulações computacionais etc. Importante ressaltar que não se trata de uma lista de exercícios repetitivos, mas preza-se pela qualidade de poucos problemas a serem investigados colaborativamente pelos grupos.

Para sistematizar por escrito as soluções dos problemas de implementação do modelo, as equipes produzem relatórios em que organizam suas respostas ao focalizar conteúdos conceituais, procedimentais e atitudinais; bem como discussões críticas. O relatório é importante como forma de incentivar a produção textual coletiva entre os integrantes do grupo colaborativo. A produção grupal dos relatórios pode 
possibilitar que os estudantes aprofundem compreensões ao terem que pesquisar e discutir sobre conceitos e sobre procedimentos que consideram relevantes de serem expostos no texto escrito. Nesse sentido, a produção do relatório tem-se mostrado relevante como gerador de debates e de reflexões entre os integrantes de uma mesma equipe, podendo favorecer profundas discussões.

Na segunda sessão de discussão, os grupos colaborativos sistematizam e defendem as soluções aos problemas de implementação do modelo, ao passo que justificam procedimentos e pensamentos com argumentos científicos. O professor assume a importante tarefa de orientar o discurso de modelagem de modo a fazer com que os estudantes insiram suas justificativas em teorias e em leis científicas. Wells, Hestenes e Swackhamer (1995) propõem como forma de motivar episódios argumentativos que os relatórios escritos sejam redistribuídos entre os grupos colaborativos de modo a fazer com que uma equipe avalie criticamente as soluções de outra equipe. Conforme as soluções dos problemas vão sendo compartilhadas e refinadas, a estrutura epistêmica do modelo matemático ganha novos significados, que podem favorecer compreensões e aplicações em novas situações.

Antes de apresentar a pesquisa de campo, resta dizer que a avaliação do ciclo de modelagem de Hestenes é formativa no decorrer de todo o processo de modelagem matemática. No entanto, o professor pode checar de alguma maneira a aprendizagem individual dos estudantes e decidir entre resolver outros problemas ou iniciar novo ciclo para o estudo de outro campo conceitual.

\section{EM CAMPO COM PROFESSORES}

Para entender como a modelagem matemática pode promover competências essenciais para a formação de sujeitos letrados cientificamente, no ano de 2016 desenvolveu-se ciclos de modelagem com 13 (treze) professores de ciências da rede municipal de educação da cidade de Almeirim, Pará, Brasil. 
O tempo de docência dos sujeitos da pesquisa variou entre 03 a 21 anos, com média de 8,8 anos e desvio padrão de 5,0 anos. Esse perfil docente foi importante para fundamentar percepções dos professores sobre as tarefas realizadas nos ciclos de modelagem. Importante ressaltar que, embora os professores já fossem atuantes no ensino fundamental, ainda não possuíam a graduação universitária, motivo pelo qual estavam cursando uma disciplina de estágio I em física como componente curricular de uma licenciatura integrada matemática-física de uma universidade pública federal pelo Plano Nacional de Formação de Professores da Educação Básica (PARFOR). Desse modo, foram desenvolvidos três ciclos de modelagem, sendo um ciclo sobre a polvição sonora na escola, um ciclo sobre o lixo de papel na sala de aula e um ciclo de modelagem sobre a obesidade entre os professores.

Conforme sintetizado no Quadro 2, o planejamento das atividades limitou-se a ações referentes ao momento de elaboração do modelo, ou seja, não foi realizado o momento de implementação do modelo. Decerto que isso permitiu a diminuição da quantidade de possíveis variáveis intervenientes e aumentou o foco na interpretação dos dados produzidos. Todavia, na continuidade das investigações, pretende-se analisar ciclos de modelagem completos com vistas à compreensão mais avançada sobre possibilidades e desafios para o letramento científico.

QUADRO 2 - Síntese das ações desenvolvidas em cada ciclo de modelagem

\begin{tabular}{|c|c|}
\hline Etapas & Ações \\
\hline \multirow{3}{*}{ Descrição do Problema. } & Apresentação motivadora do tema. \\
\cline { 2 - 2 } & Caracterização do modelo matemático. \\
\cline { 2 - 2 } Laboratório de Investigação. & Detalhamento conceitual. \\
\cline { 2 - 2 } & Planejamento da investigação. \\
\cline { 2 - 2 } & Produção de dados. \\
\hline \multirow{2}{*}{ Sessão de Discussão de modelos matemáticos. } \\
\cline { 2 - 2 } & Defesa dos modelos matemáticos. \\
\cline { 2 - 2 } & Proposta de soluções \\
\hline
\end{tabular}

Fonte: Autor (2018). 
Conforme sintetizado no Quadro 2, organizou-se as ações referentes ao estágio de elaboração do modelo em três etapas: descrição do problema, laboratório de investigação e sessão de discussão. Por sua vez, cada etapa foi subdivida em três subetapas. A etapa de descrição do problema foi organizada em apresentação motivadora do problema, caracterização estrutural do modelo matemático e detalhamento conceitual. A etapa de laboratório de investigação foi organizada em planejamento de ações, produção de dados e registro de modelos matemáticos nos whiteboards. A etapa de sessão de discussão foi organizada em defesa de modelos matemáticos, proposta de soluções e produção escrita. Ressalta-se que essas etapas e subetapas não são rígidas, evidentemente, são flexíveis e podem variar de acordo com a natureza do ambiente educacional em que se realiza o ciclo de modelagem; contudo, possibilitam planejamento prévio que pode favorecer ao desenvolvimento de competências para o letramento científico.

\section{COMPETÊNCIAS EM DESENVOLVIMENTO}

Nesta seção, o objetivo é investigar evidências que fundamentem reflexões para entender possíveis competências ao letramento científico emergentes no ciclo de modelagem. A interpretação de dados produzidos foi orientada por procedimentos da metodologia mista, em que houve enfoque tanto em técnicas qualitativas, quanto em técnicas quantitativas (CRESWELL e CLARK, 2013).

Desse modo, ao final do terceiro ciclo de modelagem, solicitou-se que os professores respondessem a um questionário fechado (Anexo I). O questionário foi composto das vinte competências do inventário de competências ao letramento científico (Quadro 1) em que os professores deveriam assinalar, a partir de suas percepções, em qual fase do ciclo de modelagem (descrição do problema; laboratório de investigação e sessão de discussão) determinada competência poderia ser desenvolvida. Sublinha-se que, antes de os professores responderem aos questionários, as vinte competências foram lidas e discutidas coletivamente para esclarecer 
quaisquer dúvidas. Após as respostas, houve roda de conversa sobre o que haviam respondido para fortalecer significados.

Assim, o corpus de análise constando de questionários fechados, de imagens, de vídeos, de transcrições e de anotações de campo foi interpretado por meio de análise textual discursiva em três momentos: unitarização, categorização e metatexto. Num primeiro instante, da unitarização, realizou-se leitura cuidadosa do material para identificar unidades de significado em cada unidade de análise.

Unitarizar um texto é desmembrá-lo, transformando-o em unidades elementares, correspondendo a elementos discriminantes de sentidos, significados importantes para a finalidade da pesquisa, denominadas de unidades de significado (MORAES e GALIAZZI, 2016, p. 71).

As unidades de significado geraram subcategorias que foram codificadas por meio de sistema alfanumérico.

Num segundo instante, da categorização, buscou-se perceber convergências/divergências de significados entre as subcategorias, reagrupando-as em categorias emergentes. Categorizar,

[...] corresponde a simplificações, reduções e sínteses de informações da pesquisa, concretizadas por comparação e diferenciação de elementos unitários, resultando em formação de conjuntos de elementos que possuem algo em comum (MORAES e GALIAZZI, 2016, p. 97).

Por fim, elaborou-se um metatexto com interlocuções empíricas e teóricas resultante de descrições e de interpretações das categorias emergentes. O metatexto representa "[...] sínteses elaboradas pelo pesquisador no sentido de expressar as novas compreensões atingidas em relação ao seu objetivo de pesquisa" (MORAES e GALIAZZI, 2016, p. 111).

A seguir, apresentam-se os resultados encontrados.

\section{Descrição do problema}

O gráfico seguinte ilustra competências identificadas pelos professores na fase de descrição do problema. 
FIGURA 3 - Competências apontadas como emergentes na fase de descrição do problema (CDP)
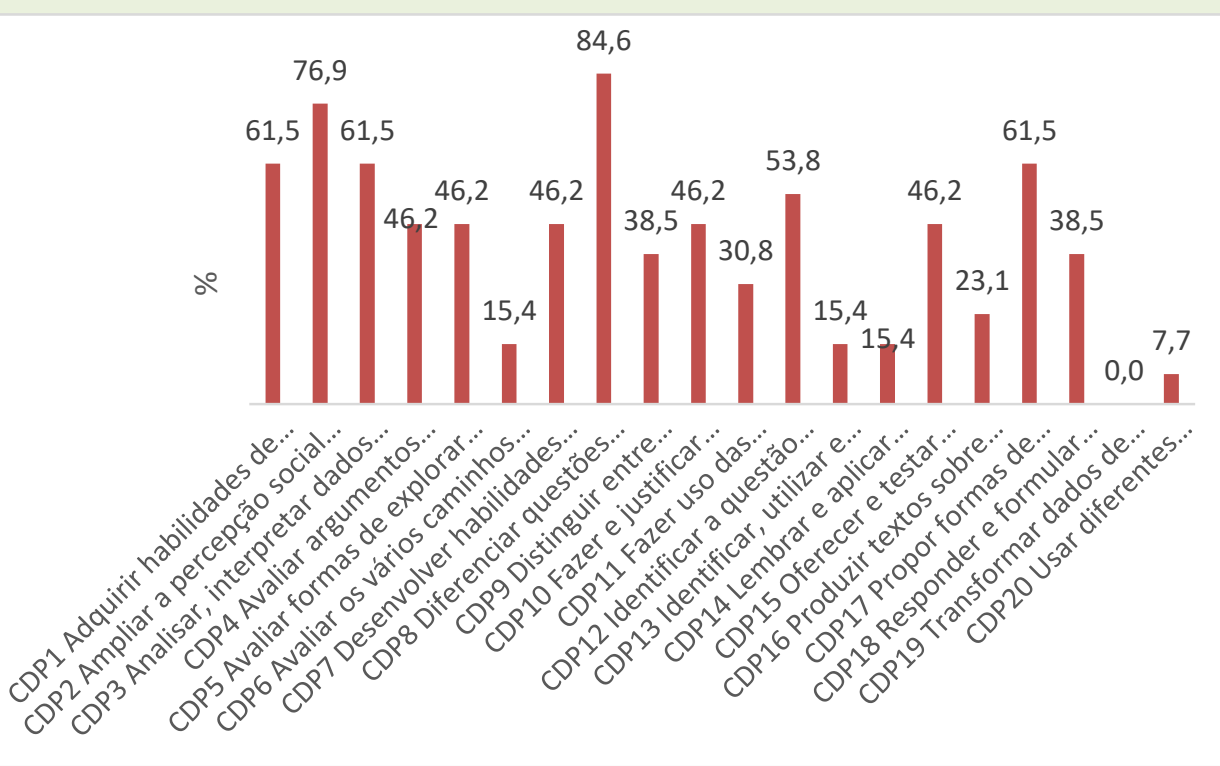

Fonte: Autor (2018).

Na Figura 3, observa-se que a competência de maior grau de força, em que o grau de força indica a maior ou menor tendência de desenvolvimento de determinada competência, foi CDP8, com taxa de 84,6\% (11 dos 13 professores). Em seguida, com médio grau de força, surgem as competências: CDP2, com taxa de 76,9\% (10 dos 13 professores); CDP1, CDP3 e CDP17, cada uma com taxa de 61,5\% (08 dos 13 professores); CDP12, com taxa de 53,8\% (07 dos 13 professores); CDP4, CDP5, CDP7, CDP10 e CDP 15 cada uma com taxa de 46,2\% (06 dos 13 professores); CDP9 e CDP 18 cada uma com taxa de 38,5\% (05 dos 13 professores); CDP16 com taxa de 23,1\% (03 dos 13 professores); CDP6, CDP13 e CDP14, cada uma com taxa de 15,4\% (02 dos 13 professores). Por fim, com baixo grau de força e com taxa de 7,7\% (01 dos 13 professores) surge a competência CDP20.

Assim, considerando o alto grau de força, para os professores participantes da pesquisa, a fase de descrição do problema parece ter sido favorável principalmente para a construção da capacidade de diferenciar questões com possibilidades de serem investigadas cientificamente, como é possível inferir na fala de um dos professores: "a escolha da questão foi 
bastante interessante, pois nela vimos o que seria ideal para trabalhar sobre - tema, a variável que direcione para que tipo de estudo você quer realizar". Para este professor, a discussão do problema foi importante para selecionar ou definir o que realmente investigar sobre o tema, ou seja, questões passíveis de serem investigadas e questões que não apresentam condições de investigação científica. Para este sujeito, o levantamento de variáveis durante a descrição do problema também foi relevante para analisar questões ideais sobre o tema de investigação.

Quanto à independência dos aprendizes para selecionar questões em ciclos de modelagem, Hestenes (2010) comenta que os estudantes que assimilam o conhecimento procedimental conseguem prosseguir de maneira sistemática em suas próprias pesquisas, sem muita dependência do professor. Reflete-se que, ao se sentirem independentes para levar a cabo suas próprias pesquisas, possivelmente, essa sensação de independência científica fez com que os professores relatassem a construção da capacidade de diferenciar questões científicas como uma das principais competências emergentes no momento da descrição do problema.

Contudo, dado o baixo grau de força, a fase de descrição do problema parece não ter sido favorável, na visão dos sujeitos da pesquisa, para a construção da capacidade de transformar dados de uma representação para outra. Sobre essa questão, um professor comenta "a dificuldade de definir as variáveis necessárias e mais importantes a serem discutidas e socializadas". Entende-se que a descrição do problema pode possibilitar aos modeladores melhorar a compreensão sobre as variáveis em jogo no ciclo de modelagem, mas nem sempre é fácil a definição dessas variáveis. Essa definição envolve descartar outras variáveis que poderiam promover discussões talvez até mais ricas que a variável escolhida de fato pelos professores.

A caracterização de variáveis, portanto, é uma capacidade que precisa ser incentivada para ser uma competência passível de ser construída no momento da descrição do problema. 


\section{Laboratório de investigação}

No seguinte gráfico, representa-se o resultado para as competências apontadas pelos sujeitos da pesquisa na fase de laboratório de investigação.

FIGURA 4 - Competências apontadas como emergentes na fase de laboratório de investigação (CLI)
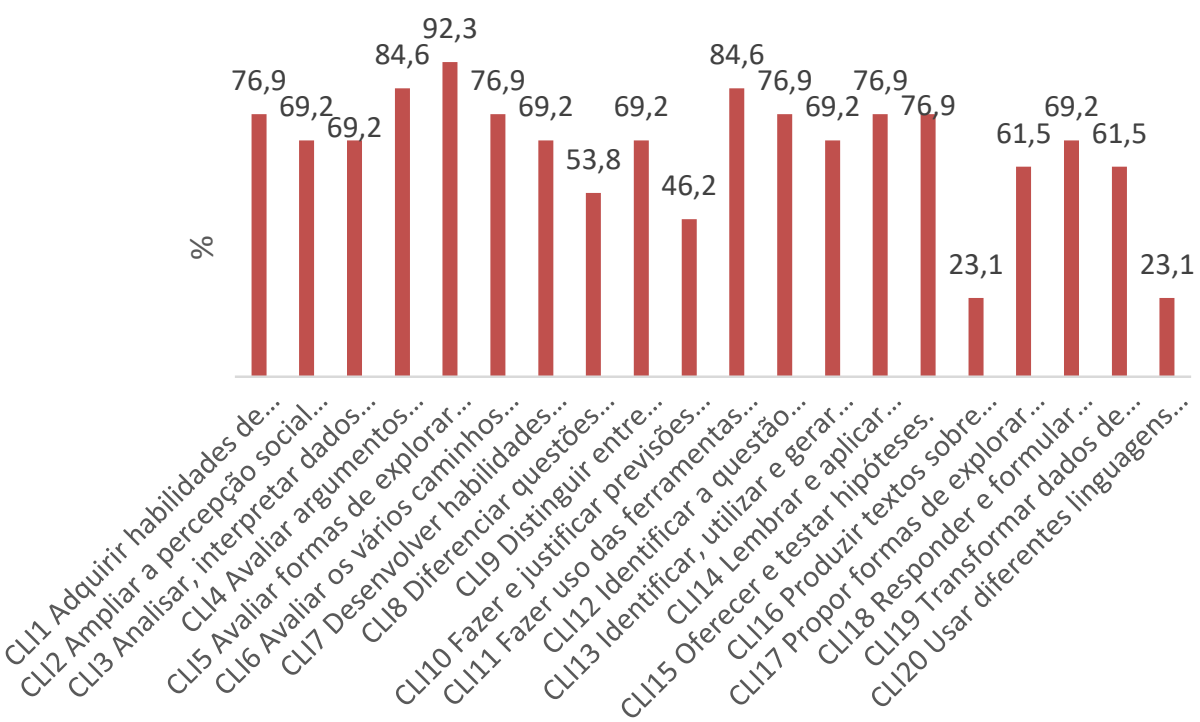

Fonte: Autor (2018).

Conforme ilustrado na Figura 4, na visão dos sujeitos da pesquisa, a competência de maior grau de força foi CLI5, com taxa de 92,3\% (12 dos 13 professores). Em seguida, com médio grau de força, surgem as competências: CLI4 e CLI11, ambas com taxa de 84,6\% (11 dos 13 professores); CLI1, CLI6, CLI12, CLI14 e CLI15, cada uma com taxa de 76,9\% (10 dos 13 professores); CLI2, CLI3, CLI7, CLI9, CLI13 e CLI18, cada uma com taxa de 69,2\% (09 dos 13 professores); CLI17 e CLI19 ambas com taxa de 61,5\% (08 dos 13 professores); CLI8 com taxa de 53,8\% (07 dos 13 professores); CLI10 com taxa de 46,2\% (06 dos 13 professores). Por fim, com baixo grau de força, cada uma com taxa de $23,1 \%$ (03 dos 13 professores), as competências CLI16 e CLI2O.

Esses dados evidenciam que, para os sujeitos da pesquisa, a fase de laboratório de investigação parece ter sido favorável, dado o alto grau de força, principalmente para a emergência da capacidade de avaliar formas de explorar cientificamente dada questão. Nessa direção, um professor comenta que "na produção de dados nós percebemos e pesquisamos as 
informações de como obtermos informações para satisfazer as nossas necessidades". Para este professor, o fato de terem que pesquisar sobre processos investigativos foi significativo para avaliar as diferentes maneiras que poderiam ser aplicadas para explorar o problema em foco.

Quanto ao processo de pesquisa, Hestenes (2010) reflete que o primeiro passo é construir um modelo explícito da situação implícita nas condições iniciais do problema. Em seguida, faz-se a extração de uma resposta para a questão proposta. Talvez em função desse processo científico próprio do ciclo de modelagem foi que os professores perceberam a potencialidade para avaliar diferentes maneiras de questionar sobre 0 problema enfrentado.

No entanto, considerando o baixo grau de força, na visão dos sujeitos da pesquisa, o laboratório de investigação parece não ter contribuído para a produção de textos científicos e para usar diferentes linguagens no decorrer da aprendizagem. Na visão de outro professor: "vejo como negativo nessa etapa a dificuldade que encontramos na absorção dos dados que, em alguns momentos, nos remeteram à pesquisa em fontes diferentes, além da sala de aula". Para este professor, houve dificuldade para absorver os dados produzidos em campo visando a elaborar o modelo matemático, o que necessitou fazer pesquisas adicionais para alcançar tal objetivo. Além disso, a dificuldade na absorção dos dados parece ter inibido o uso de diferentes linguagens no processo investigativo.

Reflete-se que os professores podem não ter percebido a capacidade de produção de texto no decorrer da fase de laboratório de investigação porque não foi solicitado explicitamente atividade desse tipo nessa etapa específica do ciclo de modelagem. Porém, chama-se a atenção para o fato de os professores não terem notado a capacidade de usar diferentes linguagens, talvez porque não ficou claro sobre quais tipos de linguagens o questionário se referia. 


\section{Sessão de discussão}

O resultado para as competências apontadas pelos sujeitos da pesquisa na fase de sessão de discussão é mostrado no gráfico que segue.

FIGURA 5 - Competências apontadas como emergentes na fase de sessão de discussão (CSD)

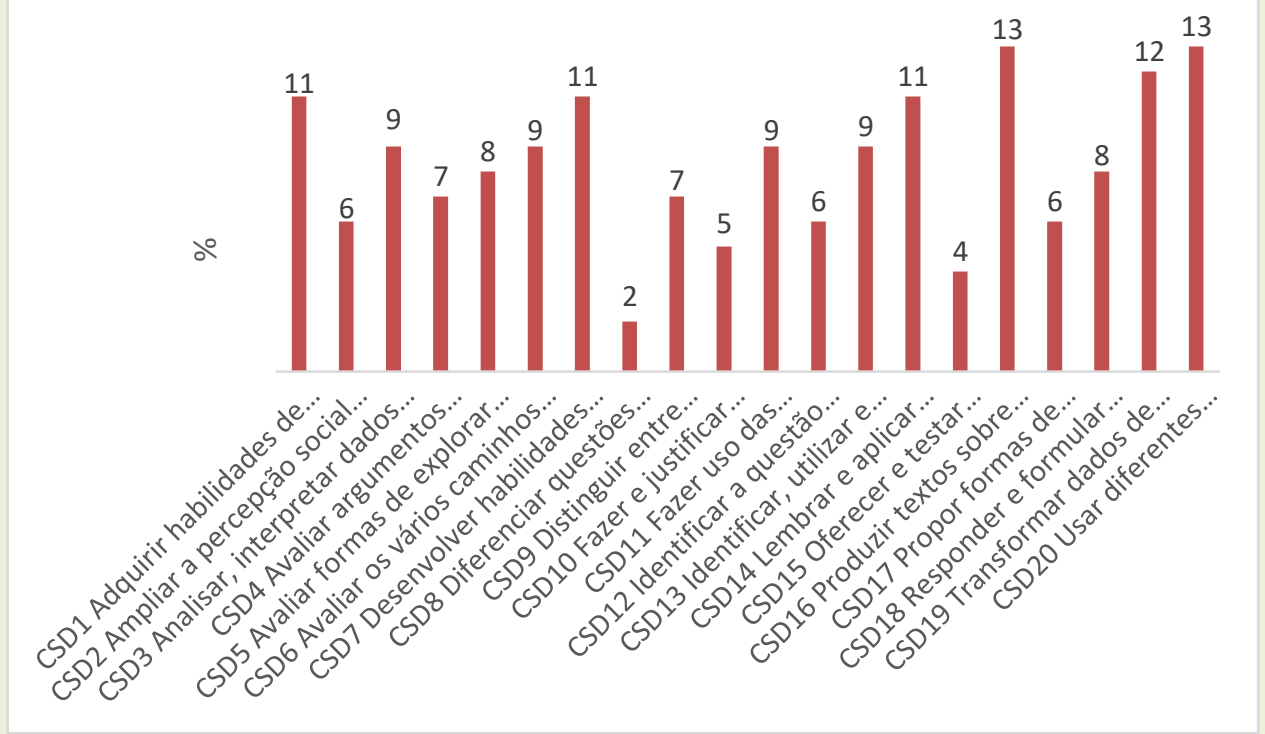

Fonte: Autor (2018).

Percebe-se na Figura 5 que as competências CSD16 e CSD20 surgem com maior grau de força, identificadas pela totalidade dos sujeitos, com taxa de $100 \%$ ( 13 dos 13 professores). Ainda com elevado grau de força, surgem as competências: CSD19, com taxa de 92,3\% (12 dos 13 professores); CSD1, CSD7 e CSD14 cada uma com taxa de 84,6\% (11 dos 13 professores). Com médio grau de força, surgem as competências: CSD3, CSD6, CSD1 1 e CSD13, cada uma com taxa de 69,2\% (09 dos 13 professores); CSD5 e CSD18, ambas com taxa de 61,5\% (08 dos 13 professores); CSD4 e CSD9 cada uma com taxa de 53,8\% (07 dos 13 professores); CSD2, CSD12 e CSD17 com taxa cada uma de 46,2\% (06 dos 13 professores); CSD10 com taxa de 38,5\% (05 dos 13 professores); CSD15 com taxa de 30,8\% (04 dos 13 professores). Por fim, com baixo grau de força, surge a competências CSD8, com taxa de 15,4\% (02 dos 13 professores).

Desse modo, em função do alto grau de força, para os sujeitos da pesquisa, a sessão de discussão foi importante principalmente para 
desenvolver competências para produção de textos argumentativos sobre temas em ciências e para usar diferentes linguagens para manifestar aprendizagens. Nessa direção, um professor comenta que "nos permite organizar os textos ou qualquer outro material que foi produzido durante o ciclo de modelagem". Em acréscimo, outro professor relata que "foi importante a troca de ideias com a turma, as informações expostas de diferentes maneiras pela turma, as discussões entre os colegas enriqueciam ainda mais o conhecimento científico sobre o tema". Para o primeiro professor, a fase de sessão de discussão foi relevante para sistematizar a pesquisa por meio de produção textual. Já para o segundo professor, essa etapa foi importante para promover discussões intergrupos e para apresentar as ideias por meio de diversos tipos de representações simbólicas.

Com relação às sessões de discussões, Hestenes (2010) assevera que é um momento em que os estudantes aprofundam aprendizagens porque estimula a avaliação e a consolidação das vivências em ciclos de modelagem anteriores. Nesse processo, como apontado pelos professores, por meio dos whiteboards é possível articular a produção de textos a processos argumentativos, o que pode possibilitar maior compreensão do conhecimento científico.

Porém, em função do baixo grau de força, para os sujeitos da pesquisa, a sessão de discussão parece não ter sido favorável para diferenciar questões possíveis de serem investigadas cientificamente. Nesse sentido, um professor comenta que "a meu ver, nessa etapa o ponto negativo se restringe justamente na seleção do tema que requer uma reflexão mais profunda, levando as considerações, as características que cercam o tema". Para este professor, houve dificuldade para selecionar pontos a serem investigados e discutidos em equipe. Tal percepção pode ser explicada pelo fato de que os questionamentos produzidos no discurso de modelagem visam a gerar situações argumentativas e não investigações propriamente ditas, embora eventualmente possa ser desencadeado um novo processo investigativo em função do processo argumentativo. 


\section{CONTORNO FINAL}

Ao resgatar o objetivo principal de pesquisa, que consistiv em analisar a modelagem matemática como geradora de competências essenciais para a formação de sujeitos letrados cientificamente no contexto do ensino amazônico de física, considera-se que tal propósito foi alcançado, ao menos parcialmente. Ele é relevante quando se observam resultados de avaliações internacionais que apontam que a maioria dos estudantes brasileiros no início do ensino médio não possuem capacidades suficientes para resolver e para tomar decisões frente a problemas simples do cotidiano.

Ressalta-se que as competências identificadas nesta pesquisa não são terminais, foram apontadas por professores em formação após participação efetiva em três ciclos de modelagem. Decerto que outros sujeitos poderiam apontar outras competências a partir de outras percepções, mas julga-se que é possível tecer algumas implicações iniciais ao ensino de física, especialmente no contexto amazônico.

Nesse sentido, o gráfico que segue mostra uma visão geral das frequências absolutas referentes às competências do inventário identificadas pelos professores em cada fase do estágio de elaboração do modelo do ciclo de modelagem de Hestenes. 
FIGURA 6 - Visão geral de competências emergentes no estágio de elaboração do modelo

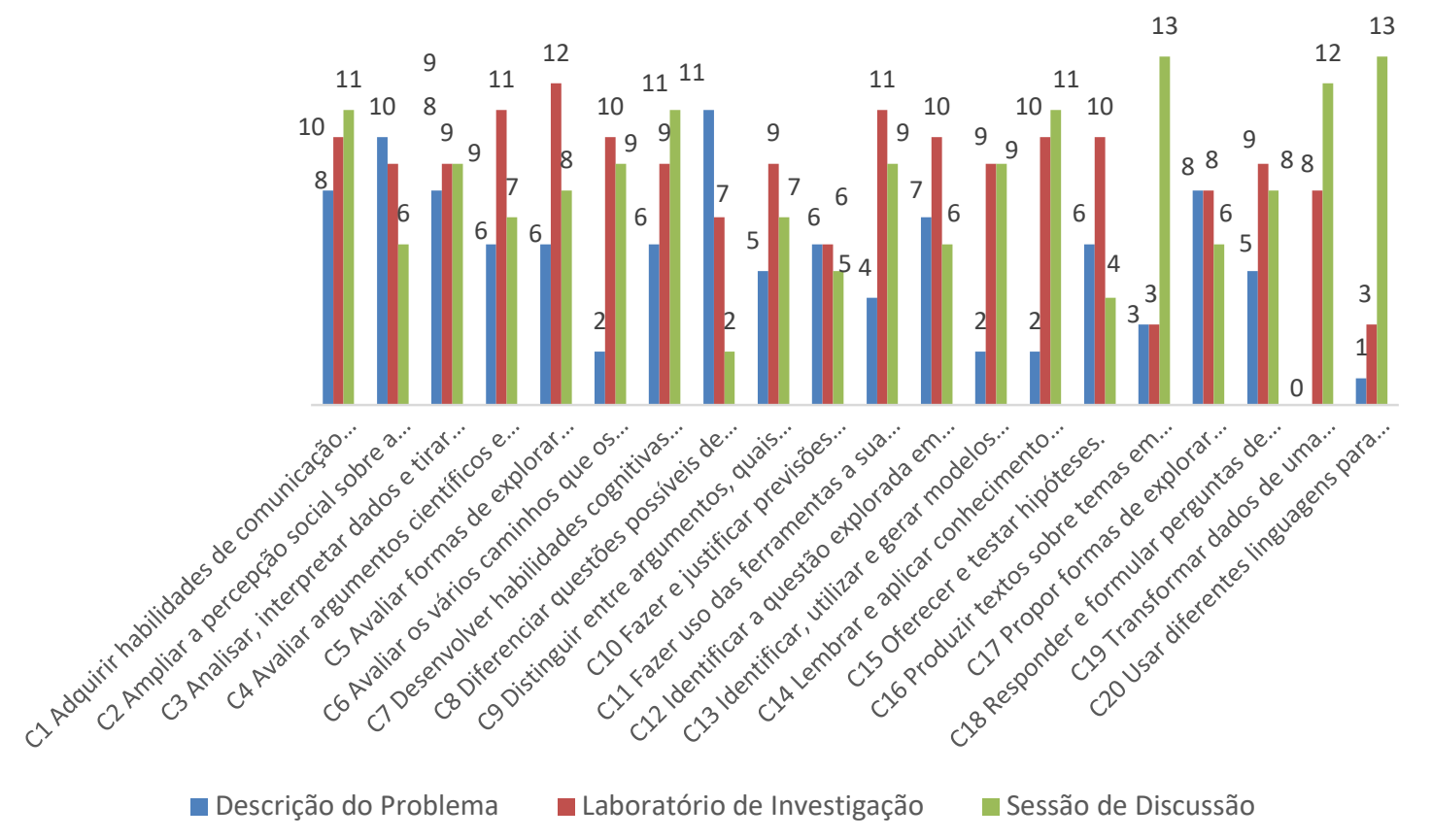

Fonte: Autor (2018).

Chama a atenção na Figura 6 que, com exceção da competência C19, todas as demais foram identificadas como possiveis de serem desenvolvidas em todas as três fases do estágio de elaboração do modelo (descrição do problema, laboratório de investigação e sessão de discussão). Portanto, é possível inferir esse estágio como potencialmente favorável ao desenvolvimento de sujeitos com capacidades básicas para o letramento científico.

A partir do evidenciado neste estudo, é possível considerar que a fase de descrição do problema mostrou-se favorável principalmente para diferenciar questões possíveis de serem investigadas cientificamente (C8). A fase de laboratório de investigação foi favorável principalmente para avaliar formas de explorar cientificamente dada questão (C12) e a fase de sessão de discussão foi favorável principalmente para produzir textos argumentativos sobre temas em ciências $(\mathrm{C} 16)$ e para usar diferentes linguagens para manifestar aprendizagens (C20).

No decorrer dos ciclos de modelagem realizados com os professores participantes da pesquisa, foi possível perceber que, quando reunidos em 
grupos colaborativos, eles parecem ter ficado mais propensos a comparar entre si as perguntas e questionamentos sobre o problema enfrentado. Esse ato de comparar as indagações pode ter levado a rejeitar perguntas consideradas "não científicas", em detrimento de perguntas com algum cunho de cientificidade, o que pode possibilitar tarefas didáticas produtivas em termos de letramento científico.

Além disso, nota-se que, ao elegerem uma pergunta considerada por eles "científica", os professores geralmente discutiam diferentes maneiras de como explorá-la durante o ciclo de modelagem. Primeiramente, por meio de conhecimentos prévios que possuíam no momento, depois, por meio de pesquisas para enriquecer seus próprios modelos mentais. A partir de novas aprendizagens potencializadas nas discussões coletivas em que se focalizava o conteúdo conceitual, procedimental e atitudinal, os professores pareciam se municiar de argumentos científicos sobre o problema enfrentado. Essa dinâmica pode, ao que parece, ao menos em algum grau, desenvolver efetivamente competências para a formação de sujeitos cientificamente alfabetizados.

Para finalizar, algumas questões surgem para investigações futuras, por exemplo, é interessante saber em que medida as competências organizadas no inventário correspondem, efetivamente, a saberes essenciais para letramento científico face ao contexto real amazônico; ou ainda saber como o inventário indicaria o desenvolvimento de letramento científico quando submetido às percepções de outros sujeitos em outros contextos educacionais brasileiros.

\section{REFERÊNCIAS}

BARSOTTI, D. C. Uso de ferramentas tecnológicas no ensino de física para o ensino médio: modelagem matemática a partir do software modellus. 2013. 90f. Dissertação (Mestrado em Ensino de Ciências Exatas). Universidade Federal de São Carlos, São Carlos/SP, 2013.

\section{BATISTA, M. C. A utilização da experimentação no ensino de física:} modelando um ambiente de aprendizagem. 2009. 85f. Dissertação (Mestrado em Educação em Ciências e Matemática). Universidade Estadual de Maringá, Maringá/PR, 2009. 
BATISTA, M. C.; KATO, L. A. Contextualização crítica nas aulas de ciências: alguns aspectos da modelagem matemática no ensino de física. In ENCONTRO PARANAENSE DE MODELAGEM EM EDUCAÇÃO MATEMÁTICA, 3, 2008, Guarapuava. Anais... Guarapuava: Universidade Estadual do Centro Oeste, 2008. p. 301-314.

BRANDÃO, R. V.; ARAÚJO, I. C.; VEIT, E. A. A modelagem científica de fenômenos físicos e o ensino de física. Física na Escola, v. 9, n. 1, 2008.

BRASIL. Ministério da Educação. Secretaria da Educação Fundamental. Parâmetros Curriculares Nacionais. Ciências Naturais. Brasília: MEC, 1997.

BRASIL. Ministério da Educação. Secretaria de Educação Básica. Orientações Curriculares para o Ensino Médio. Ciências da Natureza, Matemática e suas Tecnologias. Brasília: MEC, 2006.

BRASIL. Ministério da Educação. Secretaria de Educação Básica. Base nacional comum curricular: a educação é a base. 2018. Disponível em: http://basenacionalcomum.mec.gov.br/images/BNCC_El_EF_110518_versaofi nal_site.pdf. Acesso em 30 ago 2019.

BRASIL. Brasil no PISA 2015: análises e reflexões sobre o desempenho dos estudantes brasileiros. OCDE - Organização para a Cooperação e Desenvolvimento Econômico. São Paulo: Fundação Santillana, 2016.

CHASSOT, A. Letramento científico: questões e desafios para a educação. 6 ed. ljuí: Unijuí, 2014a.

CRESWELL, J. W.; CLARK, V., P. Pesquisa de métodos mistos. 2 ed. Porto Alegre: Penso, 2013.

FREIRE, P. Pedagogia do oprimido. 46 ed. Rio de Janeiro: Paz e Terra, 2005.

HEIDEMANN, L. A. Ressignificação das atividades experimentais no ensino de física por meio do enfoque no processo de modelagem científica. 2015. $298 \mathrm{f}$. Tese (doutorado em Ensino de Física). Universidade Federal do Rio Grande do Sul, Porto Alegre/RS, 2015.

HEIDEMANN, L. A.; ARAUJO, I. S.; VEIT, E. A. Ciclos de modelagem: uma proposta para integrar atividades baseadas em simulações computacionais e atividades experimentais. Caderno Brasileiro de Ensino de Física, v. 29, n. 2, 2012.

HEIDEMANN, L. A.; ARAUJO, I. S.; VEIT, E. A. Atividades experimentais com enfoque no processo de modelagem científica: uma alternativa para a ressignificação das aulas de laboratório em cursos de graduação em física. Revista Brasileira de Ensino de Física, v. 38, n. 1, 2016. 
HESTENES, D. O. Notes for a modeling theory of science, cognition and instruction. In: GIREP CONFERENCE, 2006, Amsterdam. Proceedings... Amsterdam: Girep, 2006, p. 34-65.

HESTENES, D. O. Toward a modeling theory of physics instruction. American Journal of Physics, v. 55, n. 5, 1987.

HESTENES, D. O. Modeling theory for math and science education. In: LESH, R. et al. (Eds.) Modeling student's mathematical modeling competencies. New York: Springer, 2010, p. 13-42.

LOZADA, C. O.; Magalhães, N. S. A importância da modelagem matemática na formação de professores de física. 2009. Disponível em:

http://www.sbf1.sbfisica.org.br/eventos/snef/xviii/atas/listaresumos.htm. Acesso em: 17 ago 2018.

MACIEL, G. S. A importância da modelagem matemática como instrumento indispensável para o ensino de física: uma reflexão aplicada ao ensino médio. 2014. 61 f. Dissertação (Mestrado em Matemática). Universidade Estadual de Santa Cruz, Santa Cruz/BA, 2014.

MENDES, J. F.; COSTA, I. F.; SOUSA, C. M. S. G. O uso do software modellus na integração entre conhecimentos teóricos e atividades experimentais de tópicos de mecânica. Revista Brasileira de Ensino de Física, v. 34, n. 1, 2012.

MONTEIRO, W. C. Modelagem matemática como meio de integração com as aulas de física. In: ENCONTRO NACIONAL DE EDUCAÇÃO MATEMÁTICA, 1 1, 2013, Curitiba. Anais... Curitiba: Pontífica Universidade Catórica, 2013, p. $01-08$.

MORAES, R.; GALIAZZI, M. C. Análise textual discursiva. 3 ed. Rio Grande do Sul: Unijui, 2016, 224p.

MOUTINHO, P. E. C. CTS e a modelagem matemática na formação de professores de física. 2007. 115F. Dissertação (Mestrado em Ensino de Ciências e Matemática). Universidade Federal do Pará, Belém/PA, 2007.

OCDE. PISA 2015 Assessment and Analytical Framework: science, reading, mathematic and financial literacy. 2016. Disponível em: https://www.oecdilibrary.org/education/pisa-2015-assessment-and-analyticalframework_9789264255425-en. Acesso em: 17 ago 2018.

PERRENOUD, P. Desenvolver competências ou ensinar saberes? a escola que prepara para a vida. Trad. Laura Solange Pereira. Porto Alegre: Penso, 2013 (Livro digital). 
PIZARRO, M. V.; LOPES JUNIOR, J. Indicadores de letramento científico: uma revisão bibliográfica sobre as diferentes habilidades que podem ser promovidas no ensino de ciências nos anos iniciais. Investigações em Ensino de Ciências, v. 20, n. 1, 2015.

SASSERON, L. H.; MACHADO, V. F. Letramento científico na prática: inovando a forma de ensinar física. São Paulo, SP: Livraria da Física, 2017.

SILVA NETO, M. J. Ensino de física pela comparação entre experimento e modelo teórico com uso da modelagem matemática. 2015. 132f. Tese (Doutorado em Educação em Ciências e Matemáticas). Universidade Federal do Pará, Belém/PA, 2015.

SOUZA, E. S. R. Modelagem matemática gerando ambiente de letramento científico: discussões no ensino de física. 2018. 237f. Tese (Doutorado em Educação em Ciências e Matemática) - Universidade Federal de Mato Grosso/Universidade Federal do Pará, Belém, 2018.

SOUZA, E. S. R. Modelagem matemática no ensino de física: registros de representação semiótica. 2010. 124f. Dissertação (Mestrado em Ensino de Ciências e Matemática) - Universidade Federal do Pará, Belém/PA, 2010.

SOUZA, E. S. R. Uma experiência com modelagem matemática para a abordagem de conceitos de física. Acta Scientiae, v. 14, n. 2, 2012.

SOUZA, E. S. R. Instrução por modelagem (modeling instruction): percepções docentes. In: ENCONTRO PARANAENSE DE MODELAGEM NA EDUCAÇÃO MATEMÁTICA. 7. 2016. Anais... Londrina: Universidade Estadual de Londrina, Londrina, 2016, p. 325-338.

SOUZA, E. S. R.; ESPÍRITO SANTO, A. O. Letramento científico em ambiente de modelagem matemática. Revista Reamec, v. 5, n. 1, 2017.

WELLS, M.; HESTENES, D. O.; SWACKHAMER, G. A. (1995). Modeling method for high school physics instruction. American JournalofPhysics, v. 63. n. 7, 1995.

WOLFF, J. F. S.; SERRANO, A. O significado da modelagem utilizada no ensino de física conforme lido a partir de referenciais da educação matemática. In: ENCONTRO NACIONAL DE PESQUISA EM EDUCAÇÃO EM CIÊNCIAS, 18, 2011, Campinas. Anais... Campinas: Universidade Estadual de Campinas, 2011 . p. $1-11$.

Agradecimentos

Agradeço à Coordenação de Aperfeiçoamento de Pessoal de Nível Superior (CAPES) código de financiamento 001.

ANEXO 


\section{Questionário fechado}

\section{QUESTIONÁRIO}

O objetivo deste questionário é analisar a emergência de competências ao letramento científico nas tarefas realizadas durante os ciclos de modelagem. Para isso, é importante que você o responda com atenção e dedicação, assinalando: quais competências você considera que emergiram durante as fases do ciclo de modelagem? Certo de sua inestimável contribuição, fica meus sinceros agradecimentos!

Prof. Ednilson Sergio Ramalho de Souza

\begin{tabular}{|l|l|c|c|c|}
\hline$N^{\circ}$ & \multicolumn{1}{|c|}{ COMPETÊNCIAS } & \multicolumn{1}{|c|}{ AÇÕES } \\
\hline 1 & Adquirir habilidades de comunicação oral e escrita. & DP & LI & SD \\
\hline 2 & $\begin{array}{l}\text { Ampliar a percepção social sobre a influência da Ciência no } \\
\text { cotidiano. }\end{array}$ & DP & LI & SD \\
\hline 3 & Analisar, interpretar dados e tirar conclusões apropriadas. & DP & LI & SD \\
\hline 4 & Avaliar argumentos científicos e evidências de diferentes fontes. & DP & LI & SD \\
\hline 5 & Avaliar formas de explorar cientificamente dada questão. & DP & LI & SD \\
\hline 6 & $\begin{array}{l}\text { Avaliar os vários caminhos que os cientistas usam para assegurar a } \\
\text { confiabilidade dos dados. }\end{array}$ & DP & LI & SD \\
\hline 7 & $\begin{array}{l}\text { Desenvolver habilidades cognitivas que permita articular o } \\
\text { conhecimento científico com a realidade vivida. }\end{array}$ & DP & LI & SD \\
\hline 8 & Diferenciar questões possíveis de serem investigadas cientificamente. & DP & LI & SD \\
\hline 9 & $\begin{array}{l}\text { Distinguir entre argumentos, quais são baseados em evidência } \\
\text { científica e quais são baseados em outras considerações. }\end{array}$ & DP & LI & SD \\
\hline 10 & Fazer e justificar previsões apropriadas. & DP & LI & SD \\
\hline 11 & $\begin{array}{l}\text { Fazer uso das ferramentas a sua disposição e do ambiente para } \\
\text { aprender ciências. }\end{array}$ & DP & LI & SD \\
\hline 12 & Identificar a questão explorada em dado estudo científico. & DP & LI & SD \\
\hline 13 & Identificar, utilizar e gerar modelos explicativos e representações. & DP & LI & SD \\
\hline 14 & Lembrar e aplicar conhecimento científico apropriado. & DP & LI & SD \\
\hline 15 & Oferecer e testar hipóteses. & DP & LI & SD \\
\hline 16 & $\begin{array}{l}\text { Produzir textos sobre temas em ciências articulando conhecimentos e } \\
\text { argumentos. }\end{array}$ & DP & LI & SD \\
\hline 17 & Propor formas de explorar cientificamente dada questão & DP & LI & SD \\
\hline 18 & Responder e formular perguntas de forma coerente. & DP & LI & SD \\
\hline 19 & Transformar dados de uma representação para outra. & DP & LI & SD \\
\hline 20 & Usar diferentes linguagens para manifestar aprendizagens. & DP & LI & SD \\
\hline
\end{tabular}

Observações:

DP: Descrição do problema; LI: Laboratório de investigação; SD: Sessão de discussão.

Recebido em: 20 de agosto de 2018 Aprovado em: 14 de maio de 2019 